\title{
Massive GI Bleed from a Marginal Ulcer Eroding into the Left Gastric Artery
}

\author{
Leopoldo M. Baccaro, MD, Kalyan Vunnamadala, MD, Uchechukwu Stanley Ogu, MD, \\ George Ibrahim, MD, Harjeet Kohli, MD, MRCP, FRCS \\ Department of General Surgery, Easton Hospital, Easton, PA, USA (Drs. Baccaro, Ogu, Ibrahim, and Kohli). \\ Division of Cardiothoracic Surgery, Baylor College of Medicine, Houston, TX, USA (Dr. Vunnamadala).
}

\begin{abstract}
Introduction: Morbid obesity is an growing problem in the United States. As the incidence of morbid obesity increases, so does the number of surgical procedures for weight loss. Surgeons must learn to manage common complications of these procedures.

Case Descriptions and Operative Technique: We present two cases of massive upper gastrointestinal hemorrhage secondary to marginal ulcers eroding into the left gastric artery. A novel surgical technique has been described from these experiences to deal quickly with this highly fatal complication.

Discussion: General surgeons must be aware of the altered anatomy and physiology because they will encounter these patients in the emergency setting. It is important to be familiar with common complications, know how to identify them in a timely manner, and treat them accordingly. Marginal ulcers are frequent; however, torrential hemorrhage secondary to these is less common and extremely lethal.
\end{abstract}

Key Words: Laparoscopic Roux-en-Y gastric bypass, Marginal ulcer after gastric bypass, Gastrointestinal bleeding, Surgical technique, Emergency surgery.

Citation Baccaro LM, Vunnamadala K, Ogu US, Ibrahim G, Kohli H. Massive GI bleed from a marginal ulcer eroding into the left gastric artery. CRSLS eCRSLS.2014.0000712. DOI: 10.4293/CRSLS.2014.0000712.

Copyright $(2014$ SLS This is an open-access article distributed under the terms of the Creative Commons Attribution-Noncommercial-ShareAlike 3.0 Unported license, which permits unrestricted noncommercial use, distribution, and reproduction in any medium, provided the original author and source are credited.

Address correspondence to: Leopoldo M. Baccaro, MD, General Surgery Resident, Department of General Surgery, Easton Hospital, 250 S. 21 st St., Easton, PA, 18042. Telephone: (610) 250-4000, Fax: 610-250-4851, E-mail: drlbaccaro@gmail.com

\section{CASE PRESENTATION 1}

A 60-year-old white woman was brought to our emergency department (ED) after being found unconscious in her home. The patient awoke and complained of severe abdominal pain and was noted to have massive hematemesis. While in our ED, the patient continued to have hematemesis and became hemodynamically unstable. Initial laboratory results revealed the following levels: hemoglobin $8.1 \mathrm{mg} / \mathrm{dL}$, lactic acid $12.7 \mathrm{mmol} / \mathrm{L}$, and creatinine $1.0 \mathrm{mg} / \mathrm{dL}$.

Initial resuscitative measures were instituted with crystalloids and vasopressors. A review of the patient's medical history was significant for open gastric bypass ( 5 years before her hospital admission), cholecystectomy, peptic ulcer disease, rheumatoid arthritis, and diabetes mellitus. The patient was also a devout Jehovah's Witness (refusing all blood products).
An urgent upper endoscopy was performed in the ED. A large marginal ulcer was noted at the anastomosis between the stomach pouch and the jejunal Roux limb. The ulcer showed a fresh clot, and when it was manipulated it produced torrential bleeding. The patient was taken urgently to the operating room for control of the bleeding.

Intraoperatively, dense intraperitoneal adhesions were found in the upper abdomen. The jejunal Roux limb was grossly dilated as was the remnant stomach. A large marginal ulcer was present at the anastomosis between the stomach pouch and the jejunal Roux limb. Exsanguinating bleeding was present from a large defect in the left gastric artery caused by the marginal ulcer. By the time control of the arterial perforation had occurred, the patient's hemoglobin level was $5 \mathrm{mg} / \mathrm{dL}$ and her systolic blood pressure was $50 \mathrm{~mm} \mathrm{Hg}$. It was apparent that the patient had 
sustained a nonsurvivable hemorrhage. She was transferred to the recovery room, and the family agreed to the termination of all supportive measures.

\section{CASE PRESENTATION 2}

A 67-year-old white man was brought to our ED after being found unresponsive at home. The previous evening he had been complaining of severe upper abdominal pain. His medical history was significant for hypertension, hyperlipidemia, and tobacco abuse. The patient also had laparoscopic gastric bypass surgery; however, he adamantly denied ever having any surgery performed.

When aroused, he was noted to have two episodes of massive hematemesis, yet he remained hemodynamically stable. His hemoglobin level was $12.8 \mathrm{mg} / \mathrm{dL}$ and creatinine level was $0.99 \mathrm{mg} / \mathrm{dL}$. A computed tomography scan was performed and revealed pancreatic inflammation; however, amylase and lipase levels were within normal limits. It also showed a staple line in the upper abdomen, suggestive of prior gastric bypass surgery. The patient was subsequently transferred to the intensive care unit and was immediately transfused with 2 units of packed red blood cells and given intravenous fluid boluses.

An immediate endoscopy was performed, which revealed an ulcer at the gastrojejunal anastomosis with no active bleeding. A blind epinephrine injection was attempted, causing the ulcer to begin bleeding torrentially. At this time, the general surgery department was called emergently and the patient was taken to the operating room.

Exploratory celiotomy revealed that the patient had undergone a prior retrocolic Roux-en-Y gastric bypass. Fortunately, this patient did not have many adhesions, and visualization of the anastomotic site was facilitated after mobilization of the gastric remnant. Both the jejunal Roux limb and the gastric pouch were extremely distended. After detaching the anastomosis, a significant amount of blood was evacuated from the intestinal tract and a large marginal ulcer on the posterior wall of the jejunum was found. This had eroded into the left gastric artery. The artery was suture-ligated, and both ends of the detached anastomosis were closed. At this point, the patient had a systolic blood pressure of $50 \mathrm{~mm} \mathrm{Hg}$ and was receiving vasopressors, so it was opted to delay the re-anastomosis and take the patient to the intensive care unit for recovery.

\section{OPERATIVE TECHNIQUE}

The operative technique described was that used for patient 2 (Figure 1). Essentially, the same steps were per- formed on both patients; however, there are minor variations to the technique when dealing with a retrocolic retrogastric Roux limb versus an antecolic Roux limb because the gastric remnant did not need to be mobilized in the latter case. Next, we describe the surgery on a patient with a retrocolic retrogastric Roux limb.

After adequate placement of the patient in the supine position, the appropriate surgical prep and draping was performed. An incision was made vertically in the midline from the xiphoid to just above the umbilicus. The skin and subcutaneous tissue were divided. The fascia and peritoneum were then opened, revealing the intraabdominal organs. The xiphoid process was excised for proper visualization. An upper bar and Balfour retractor were used to maintain exposure. The left triangular ligament was divided, and the left lobe of the liver was retracted laterally. The defunctionalized gastric remnant was mobilized to the right and the retrocolic retrogastric Roux limb was visualized. The jejunal limb and the gastric pouch were extremely distended with blood. Because of the anatomical distortion of the area, the left gastric artery was difficult to observe. Its position was posterior in relation to the gastric pouch, making access to the vessel particularly problematic. The gastrojejunal anastomosis was therefore partially disconnected, and a large amount of clotted blood was evacuated from the intestinal tract. After some suctioning, a brisk-bleeding left gastric artery was visualized. Several figure-of- 80 silk sutures were placed to ligate the bleeding left gastric artery. After ensuring that the bleeding had been controlled and the patient had been stabilized hemodynamically, surgery continued. The gastrojejunal anastomosis, which had only been taken down partially, was then completely transected using a gastrointestinal anastomosis stapler. Once freed, the mesentery of the Roux limb was divided and delivered through the window in the transverse mesocolon. It was then attached to the transverse mesocolon with several interrupted stitches using 3-0 Prolene (Ethicon, Somerville, New Jersey, USA) sutures for later reconstruction. The end of the gastric pouch had a significant opening, with a large ulcer on the posterior wall. The ulcer was excised and the wound edges freshened, and the remaining opening was closed using 2-0 Polydioxanone (PDS) in a continuous fashion. This suture was then returned to oversew the first layer. Integrity of the closure was determined by infusing the gastric pouch with dilute methylene blue. In view of the critical state of the patient, a re-anastomosis of the Roux limb to the gastric pouch was not performed and was left to be reconstructed in a later setting. 


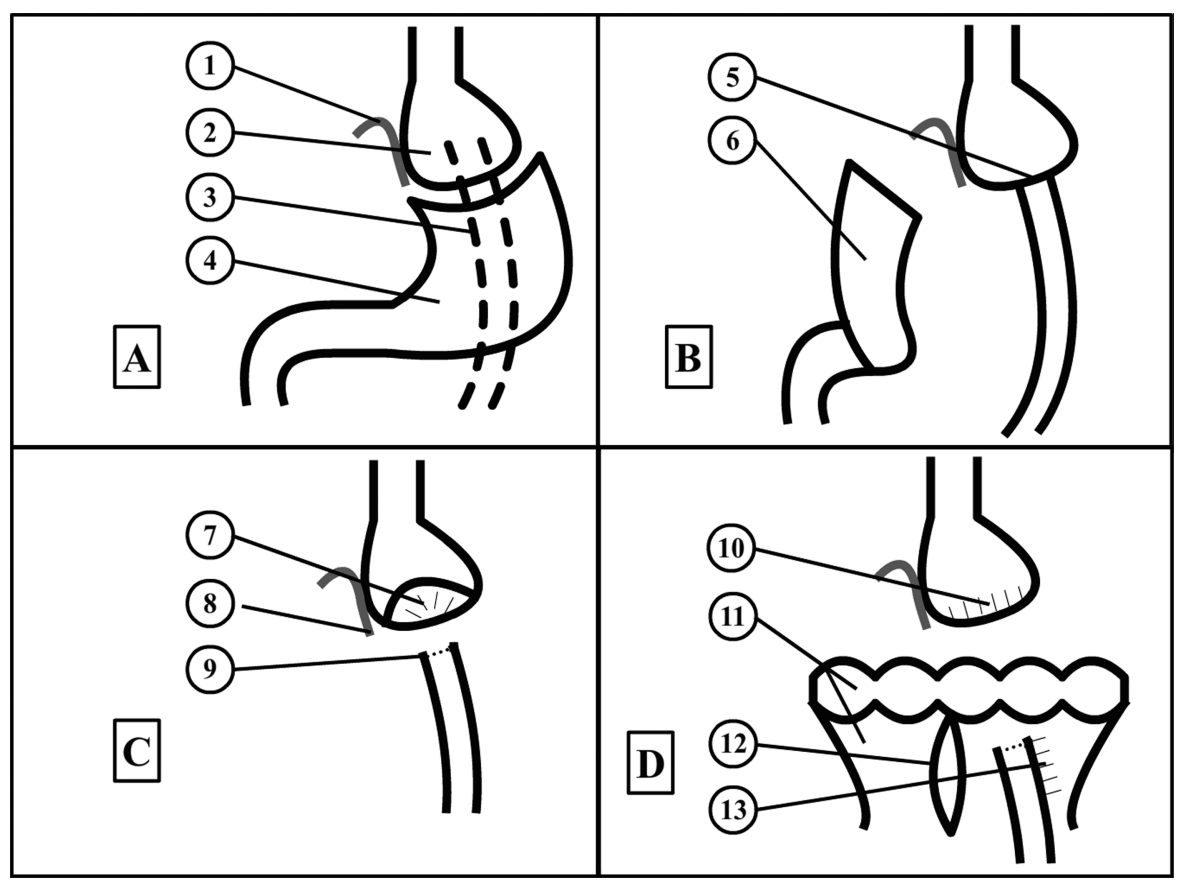

Figure 1. Schematic of the surgical technique. (A) Anatomy of Roux-en-Y gastric bypass: 1, Left gastric artery; 2, gastric pouch; 3, Roux limb; 4, gastric remnant. (B) Gastric remnant reflected to the right, revealing the gastrojejunal anastomosis: 5, gastrojejunal anastomosis; 6, reflected gastric remnant. (C) Detached gastrojejunal anastomosis: 7, marginal ulcer; 8 , suture ligation of the left gastric artery; 9, gastrointestinal anastomosis stapler across the Roux limb. (D) Finished reconstruction before closure: 10, Polydioxanone (PDS) oversewn closure of the gastric pouch; 11, transverse colon and transverse mesocolon; 12, mesocolonic defect; 13, attachment of Roux limb to the transverse mesocolon using interrupted Prolene sutures. (Image created using Adobe Flash Professional CS 6).

After several days in the intensive care unit, the patient was stabilized and taken back to the operating room for open reconstruction. This time, an antecolic approach was used. A stapled anastomosis was created between the gastric pouch and the Roux limb, and both appeared healthy and viable. The remaining defect was closed using 2-0 polyester sutures in a continuous fashion. The mesenteric defect was also closed using the same suture material. After a lengthy recovery, the patient was finally discharged home and is now fully recovered.

\section{DISCUSSION}

The number of morbidly obese individuals is reaching staggering levels. As of $2002,5.1 \%$ of the US population had a body mass index of 40 or greater. With the increased prevalence of morbid obesity, there has been an increase in the number of surgical procedures being performed. In the United States alone, bariatric surgical procedures have risen from 13,365 in 1998 to 72,177 in 2002, and to 124,838 cases in 2008.1,2 As the number of surgical procedures increases, so does the number of complications. Gastric bypass surgery is the most common bariatric surgical procedure, and the altered anatomy and physiology needs to be understood. Upper gastrointestinal bleeding in patients with gastric bypass is a potentially fatal complication and needs to be addressed in a timely fashion.

The most common cause of postoperative bleeding in patients who have undergone Roux-en-Y gastric bypass is marginal ulceration at the gastrojejunal anastomosis. ${ }^{3}$ Incidence of marginal ulcers after Roux-en-Y gastric bypass has been noted to be as high as 7\%.4 Although the gastric pouch has less acid production postoperatively, the preserved vagus nerve enables the remnant stomach to maintain an acidic environment. A long gastric pouch may increase the risk of marginal ulcer formation. Patient factors such as smoking, alcohol use, nonsteroidal anti-inflammatory drug use, diabetes, and Helicobacter pylori infections also increase the risk. ${ }^{5}$ In our first patient, we knew that none of these factors were present. In fact, an upper endoscopy performed 1 month earlier had shown no abnormalities, and we could not identify a definitive cause of how this marginal ulcer evolved so rapidly. Our second patient had a history of tobacco abuse. 
There have also been case reports of gastric bands causing left gastric artery erosion. ${ }^{5}$ Gastric band erosion has been noted to occur at a rate of $0.3 \%$ to $1.1 \% .{ }^{6}$ There is speculation that the left gastric artery may be included within a laparoscopic gastric band during initial surgery, and erosion of the band through the left gastric artery can lead to torrential hemorrhage.

Gastrogastric fistulas are also a known cause of gastrointestinal bleeding in patients with Roux-en-Y gastric bypass. Increased acid exposure from the gastric remnant to the pouch may increase the propensity of marginal ulcer formation. An ulcer eroding through the pouch toward the remnant may cause a fistula as well.

Other sources of bleeding can occur in the remnant stomach (antrum) and duodenal regions. Localization of bleeding can be done with a technetium Tc99m red blood cell scan, celiac angiogram, or intraoperative gastrotomy with subsequent endoscopic evaluation. ${ }^{6}$

Reports of perforated marginal ulcers after Roux-en-Y gastric bypass are described as well. Risk factors included steroid use, nonsteroidal anti-inflammatory drug use, smoking, and prior marginal ulcers. ${ }^{7}$

We report two unusual cases of massive upper gastrointestinal hemorrhage caused by a marginal ulcer eroding into the left gastric artery. Our first patient had refused any blood products, and this made resuscitation even more difficult. In addition, intraoperative localization of the bleeding was complicated by her dense intraperitoneal adhesions. Our second patient survived and was discharged home after a prolonged hospital course. Because of the advantage of fewer adhesions from his laparoscopic procedure, localization of the bleeding in his case was more straightforward.

Although endoscopic treatment may be attempted at first, surgical exploration is inevitable. Angiography and embolization may be an acceptable approach in a stable patient; however, the two patients we encountered showed evidence of shock and required immediate surgical intervention. As a community hospital, we also lack in-house interventional radiology staff on call, and attempting this approach would have delayed patient care. In both cases, the Roux limb was detached from the gastric pouch to expose the ulcerated mucosa and bleeding vessel. Hemostasis was achieved by suture ligation of the left gastric artery. A re-anastomosis was not attempted in the same setting because of the shock state of the patients; it was deemed not prudent to perform such a surgery in this extreme circumstance, also taking into account the increased risk of anastomotic failure in unstable and hypotensive patients.

\section{CONCLUSION}

Marginal ulcer erosion into the left gastric artery is a rare and potentially fatal complication noted after gastric bypass surgery. There has been evidence that this complication can occur in both open and laparoscopic weight loss procedures. When patients with prior Roux-en-Y gastric bypass surgery present with massive hematemesis, this condition should be considered in the differential diagnosis. Options for treatment will require operative exploration, and an upper endoscopy can be performed intraoperatively.

There has been an increasing trend in bariatric surgeries in the past decade. Both laparoscopic and general surgeons will need to intervene more frequently in serious complications secondary to Roux-en-Y gastric bypass surgery. It is important to diagnose and treat these conditions quickly to appropriately care for our patients and prevent further morbidity and mortality.

\section{References:}

1. Santry HP, Gillen DL, Lauderdale DS. Trends in bariatric surgical procedures. JAMA. 2005;294(15):1909-1917.

2. Nguyen NT, Masoomi H, Magno CP, Nguyen XM, Laugenour $\mathrm{K}$, Lane J. Trends in use of bariatric surgery, 2003-2008. J Am Coll Surg. 2011;213(2):261-266.

3. Yang CS, Lee WJ, Wang HH, Huang SP, Lin JT, Wu MS. Spectrum of endoscopic findings and therapy in patients with upper gastrointestinal symptoms after laparoscopic bariatric surgery. Obes Surg. 2006;16:1232-1237.

4. Rasmussen JJ, Fuller W, Ali MR. Marginal ulceration after laparoscopic gastric bypass on gastric resection. Am J Surg. 1976;131:162-168.

5. Azagury DE, Abu Dayyeh BK, Greenwalt IT, Thompson CC. Marginal ulceration after Roux-en-Y gastric bypass surgery: characteristics, risk factors, treatment, and outcomes. Endoscopy. 2011;43(11):950-954.

6. Zerey M, Sigmon L, Kuwada T, Heniford T, Sing R. Bleeding duodenal ulcer after Roux-en-Y gastric bypass surgery. $\mathrm{J} \mathrm{Am}$ Osteopath Assoc. 2008;108:25-27.

7. Png KS, Rao J, Lim KH, Chia KH. Lap-band causing left gastric artery erosion presenting with torrential hemorrhage. Obes Surg. 2008;18(8):1050-1052.

8. Spivak H, Favretti F. Avoiding postoperative complications with the LAP-BAND system. Am J Surg. 2002;184:31S-37S.

9. Felix EL, Kettelle J, Mobley E, Swartz D. Perforated marginal ulcers after laparoscopic gastric bypass. Surg Endosc. 2008; 22(10):2128-2132. 